\title{
A novel mutation in NDUFB11 unveils a new clinical phenotype associated with lactic
}

acidosis and sideroblastic anemia

Alessandra Torraco ${ }^{1}$, Marzia Bianchi ${ }^{1}$, Daniela Verrigni ${ }^{1}$, Vania Gelmetti ${ }^{2}$, Lisa Riley ${ }^{3,4}$, Marcello Niceta $^{5}$, Diego Martinelli ${ }^{6}$, Arianna Montanari ${ }^{7}$, Yiran Guo ${ }^{3}$, Teresa Rizza ${ }^{1}$, Daria Diodato ${ }^{1}$, Michela Di Nottia ${ }^{1}$, Barbarella Lucarelli ${ }^{8}$, Francesco Sorrentino ${ }^{9}$, Fiorella Piemonte ${ }^{1}$, Silvia Francisci $^{10}$, Marco Tartaglia ${ }^{5}$, Enza Maria Valente ${ }^{2,11}$, Carlo Dinisi-Vici ${ }^{6}$, John Christodoulou ${ }^{3,4,12}$, Enrico Bertini ${ }^{1 \#}$, Rosalba Carrozzo ${ }^{1 \#^{*}}$

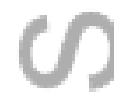

${ }^{1}$ Unit of Muscular and Neurodegenerative Diseases, Laboratory of Molecular Medicine, Bambino Gesù Children's Hospital, IRCCS, Rome, Italy; ${ }^{2}$ Neurogenetics Unit, CSS-Mendel Laboratory, IRCCS Casa Sollievo della Sofferenza, San Giovanni Rotondo, Italy; ${ }^{3}$ Genetic Metabolic Disorders Research Unit, Children's Hospital at Westmead 2145, Sydney, Australia; ${ }^{4}$ Discipline of Paediatrics \& Child Health, University of Sydney 2006, Sydney, Australia; ${ }^{5}$ Division of Genetic Disorders and Rare Diseases, Bambino Gesù Children's Hospital, IRCCS, Rome, Italy; ${ }^{6}$ Division of Metabolism, Bambino Gesù Children's Hospital, IRCCS, Rome, Italy; ${ }^{7}$ Pasteur Institute - Cenci Bolognetti Foundation, Sapienza University of Rome, Rome, Italy; ${ }^{8}$ Stem Cell Transplant Unit, Department of Hematology and Oncology, Bambino Gesù Children's Hospital, IRCCS, Rome, Italy; ${ }^{9}$ UO Talassemici -Anemie Rare del Globulo Rosso, Ospedale S Eugenio, Rome, Italy; ${ }^{10}$ Department of Biology and Biotechnologies "C. Darwin", Sapienza University of Rome, Rome, Italy; ${ }^{11}$ Section of Neurosciences, Department of Medicine and Surgery, University of Salerno, Salerno, Italy;

${ }^{12}$ Discipline of Genetic Medicine, University of Sydney 2006, Sydney, Australia.

This is the author manuscript accepted for publication and has undergone full peer review but has not been through the copyediting, typesetting, pagination and proofreading process, which may lead to differences between this version and the Version of Record. Please cite this article as doi: $10.1111 /$ cge. 12790

This article is protected by copyright. All rights reserved. 
\#These authors contributed equally as senior authors

${ }^{*}$ Corresponding Author

Rosalba Carrozzo

Unit of Muscular and Neurodegenerative Disorders,

Laboratory of Molecular Medicine, “Bambino Gesù” Children’s Hospital, IRCCS,

Viale di San Paolo, 15 - 00146 Rome, Italy;

phone: +390668593599; Fax: +390668592024; e-mail: rosalba.carrozzo@opbg.net

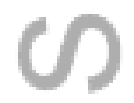

Conflict of interest

All authors declare that there is no conflict of interest.

\section{Acknowledgments}

This work received financial support from the Telethon Grant GGP11011, the Italian Ministry of Health (GR2010-2316392), and the Italian Association of Mitochondrial Disease Patients and Families (Mitocon). This research was also supported by Australian NHMRC grant 1026891 to J.C., and we also gratefully acknowledge donations to J.C. by the Crane and Perkins families. 


\begin{abstract}
NDUFB11, a component of mitochondrial complex I, is a relatively small integral membrane protein, belonging to the 'supernumerary' group of subunits, but proved to be absolutely essential for the assembly of an active complex I. Mutations in in the X-linked nuclear encoded NDUFB11 gene have recently been discovered in association with two distinct phenotypes, i.e. microphthalmia with linear skin defects and histiocytoid cardiomyopathy. We report on a male with complex I deficiency, caused by a de novo mutation in NDUFB11 and displaying early onset sideroblastic anemia as the unique feature. This is the third report that describes a mutation in NDUFB11 but all are associated to a different phenotype. Our results further expand the molecular spectrum and associated clinical phenotype of NDUFB11 defects.
\end{abstract}

\title{
Keywords
}

Mitochondrial disease, NDUFB11, OXPHOS, sideroblastic anemia

This article is protected by copyright. All rights reserved. 


\section{Introduction}

Mitochondrial diseases are due to a reduced capacity of oxidative phosphorylation and, in a large proportion of cases, the problem can be due to a complex I defect (CI) (MIM \#252010) (1). The mammalian CI (NADH-CoQ oxidoreductase) is a multisubunit complex that catalyzes the first step in the electron transport chain, transferring 2 electrons from NADH to ubiquinone coupled to the translocation of 4 protons across the membrane (2). It consists of 44 subunits thus resulting in the largest enzyme of the oxidative phosphorylation (OXPHOS) system. Fourteen out of the 44 subunits constitute the "catalytic core" $(3,4)$, the remaining subunits are considered "ancillary" subunits, and their role in the assembly, stabilization, and activity of the complex is still under active investigation, although it seems that some of them are absolutely essential for the assembly and stabilization of an active CI $(5,6)$.

Complex I deficiency is the most frequently observed cause of OXPHOS disorders, varying widely in the severity of symptoms and clinical presentations. Typical clinical presentations include cardiomyopathy, pure myopathy, leukoencephalopathy, hepatopathy with tubulopathy, and most commonly Leigh or Leigh-like syndromes $(7,8,6,9)$. Hematological manifestations including aplastic, macrocytic, or sideroblastic anemia, leukopenia, neutropenia, thrombocytopenia, or pancytopenia can also occur (10). Clinical phenotypes can have early or delayed onset, with the former generally followed by rapid progression and death within a few years. Due to the size of CI, the dual genetic origin of its subunits and the high number of ancillary proteins involved in its assembly, the identification of causative gene mutations is often challenging. Next Generation Sequencing (NGS) has been proved to be a powerful tool to find new genes involved in CI defect and provide a molecular definition for undiagnosed patients. 
In the last year, mutations have been discovered by two different groups in NDUFB11, a gene located in the short arm of the X-chromosome (Xp11.23). The first group described two de novo variants in two unrelated females affected by microphthalmia with linear skin defects (11), while the second group described one de novo mutation in one female patient and a X-linked inherited heterozygous 1-bp deletion in a second individual, associated with histiocytoid cardiomyopathy (12). NDUFB11 is a relatively small integral membrane protein (122 amino acids), which belongs to the 'supernumerary' group of subunits, but proved to be essential for the assembly of an active complex I $(13,11)$.

We here report the clinical, biochemical, and molecular characterization of one patient with lactic acidosis, sideroblastic anemia and isolated defect of complex I due to a de novo threenucleotide deletion (c.276_278delCTT) in NDUFB11 gene.

\section{Materials and Methods}

\section{Case report}

The study was approved by the Ethical Committee of the Children Hospital Bambino Gesù, Rome, Italy, in agreement with the Declaration of Helsinki. Informed consent was signed by the parents of the patient.

The patient is a boy born from healthy unrelated parents at 38 weeks of gestation by planned caesarean delivery because a persistent fetal tachycardia was noticed during the last month of gestation. Oligohydramnios and fetal ventricular hypertrophy were detected by ultrasound examination during antepartum surveillance. At birth, weight was $2.9 \mathrm{~kg}$ and heart involvement was confirmed by the finding of an aneurysm of the fossa ovalis associated to hypertrophy of the ventricular walls with moderate trabeculature of the right ventricle (interventricular septum $=5.6$ 
$\mathrm{mm}$ ), along with mild anemia ( $\mathrm{Hb} 10.4 \mathrm{~g} / \mathrm{dl})$. In the first months of life, hemoglobin was low (7 g/dl) and unresponsive to vitamin B6, B12 and folic acid. At the age of 3 months the child was admitted to the "Bambino Gesù" Children's Hospital because of worsening of his general conditions and failure to thrive. Physical examination showed mild dysmorphic features (hypertelorism, saddle nose, low set ears), systolic murmur at the centrum of $2 / 6$, hepatosplenomegaly and hydrocele. Routine blood tests revealed severe anemia without a hemolytic component ( $\mathrm{Hb}$ : 5.9-6.3 g/dl; hematocrit: 19.0\%, nv 30.00-45.00; red blood count: 2,20x10x6/uL; MCV: 91.6 fl, nv 70.00-86.00; RET\%: 2.68, nv 0.20-2.00; reduced haptoglobin), metabolic acidosis (EAB $7.1 \mathrm{mEq} / 1, \mathrm{NaHCO} 317.9 \mathrm{mEq} / \mathrm{l})$, and hyperlactacidemia (4.2-8.2 mmol/1, nv $<2.1$ ). Blood levels of vitamin B12 folates, iron and ferritin were in the normal range. The peripheral smear showed hypochromic red cells with anisopoikilocytosis, and normal white blood cell morphology. Bone marrow aspiration documented nonspecific abnormalities, with a myelogram represented by $13 \%$ of erythroblasts with notes of dyserythropoiesis, $30 \%$ granuloblasts, $41 \%$ of mononuclear elements and $16 \%$ of no-granulocyte undifferentiated elements. Virology studies for Cytomegalovirus, Parovirus B19 and Epstein-Barr virus were negative. No cyogenetic abnormalities were found. Flow cytometry showed immaturity of B lymphocytes. Congenital defects of erythrocyte glucose-6-phosphate dehydrogenase and pyruvate kinase were formally excluded, as well as congenital hemoglobinopathies. Erythrocyte osmotic resistance was also in the normal range. Treatment with folic acid, iron and erythropoietin was undertaken, without any benefit, and the patient required regular transfusional therapy with red cells from the age of 5 months.

A subsequent bone marrow biopsy was performed at 12 months of age due to persistent anemia requiring increasing need of packed red cell transfusions. On a marrow stained with Prussian blue, 
ring sideroblasts (99\%) were detected, and notes of myelodisplasia were described, in the absence of cyogenetic abnormalities. The presence of ring sideroblasts, together with the clinical history, led to make the diagnosis of sideroblastic anemia, a form of anemia in which the heme synthesis or processing in the bone marrow is dysfunctional. Granular deposition of iron in the mitochondria forms a ring around the nucleus of the developing red blood cells, producing ringed sideroblasts rather than healthy eritrocytes. Metabolic workout showed a persistent hyperlactacidemia, with increased alanine (436 $\mu \mathrm{mol} / \mathrm{l}$, nv 200-450) in the blood amino acid profile, together with high levels of lactic acid and Krebs cycle intermediates in urinary organic acids. Motor milestones were reportedly normal and the child was able to walk independently at the age of 15 months. Language development was also normal. Skin and muscle biopsies were performed around the age of 2 years to investigate the hypothesis of mitochondrial disease. Histological and histochemical study of the quadriceps muscle biopsy specimen showed mild diffuse aspects of mitochondrial proliferation.

The patient is in follow-up and needs transfusions every two weeks. The iron chelation therapy with deferasirox has been started when the patient was 7 years old, and the liver iron concentration, periodically measured by Superconducting Quantum Interference Device (SQUID), results in the normal range. Besides persistent hematological problems, motor and cognitive abilities remain normal. He attends regular school with good marks and has never suffered from neurological defects. A brain MRI at age 9 years showed no abnormalities.

\section{Biochemical studies}

Mitochondrial respiratory chain (MRC) complex activities were measured in muscle biopsy using a reported spectrophotometric method (14). 
Human fibroblasts were obtained from skin biopsies and grown in DMEM medium supplemented with $10 \%$ foetal bovine serum, $4.5 \mathrm{~g} / \mathrm{L}$ glucose, $50 \mu \mathrm{g} / \mu \mathrm{l}$ uridine.

Complex V activity (in the direction of ATP synthesis) was measured in fibroblast mitochondria of the patient and age-matched controls, using reported spectrophotometric methods (15). Either succinate (the direct substrate of complex II-succinate dehydrogenase), or malate (which is utilized by malate dehydrogenase to generate $\mathrm{NADH}$, substrate of complex I-NADH dehydrogenase) were used as substrates. Complex I and IV activities were also measured in $15 \mu \mathrm{g}$ cleared cell lysates of patient and age-matched control fibroblasts using dipstick enzyme activity assays according to the manufacturer's instructions (Mitosciences, Eugene, Oregon, USA).

For blue native gel electrophoresis (BNGE) either fibroblasts or fibroblast-derived mitochondria, from controls and patient, were processed according to Nijtmans procedures (16). Briefly, $2.5 \times 10^{6}$ fibroblasts were collected and resuspended in $200 \mu$ of 1 XPBS. Digitonin was titrated and added at a concentration of $2.8 \mu \mathrm{g} / \mu \mathrm{l}$. After 10' incubation on ice, samples were centrifuged at $21000 \mathrm{xg}$ for 5, and supernatant was discarded. Either digitonin pellet or fibroblast mitochondria were resuspended in 1.5 M aminocaproic acid, $50 \mathrm{mM}$ Bis-Tris ( $\mathrm{pH} 7.0)$ and solubilized with $1 \%(\mathrm{v} / \mathrm{v})$ of lauryl maltoside (Sigma Aldrich). Thirty to sixty micrograms proteins were separated by BNGE in a linear 5-13\% gradient gel and subjected either to Western blotting or in-gel activity assay (17). For electrophoresis in SDS polyacrylamide gels (SDS-PAGE), $30 \mu \mathrm{g}$ of fibroblast mitochondria were loaded in a $12 \%$ denaturing gel.

For immunoblot analysis of OXPHOS complexes, PVDF membranes were probed with monoclonal antibodies purchased from Mitosciences (Eugene, OR, USA) and recognizing the following proteins: complex I - 39kDa subunit (NDUFA9); complex II - 70kDa (SDHA); complex III UQCRC2 (Core protein 2); complex IV - subunit I and subunit II (COXI; COXII); and VDAC 
(porin) protein, the latter used as internal control for equal loading. NDUFB11 antibody was purchased from Proteintech (San Diego, CA, USA). Reactive bands were detected using the Lite Ablot Extend Long Lasting Chemiluminescent Substrate (Euroclone, Pero, Italy). Densitometry analysis was performed using the Quantity One Software (BioRad, Hercules, CA, USA).

For all experiments, age-matched controls were used.

\section{Molecular analysis}

Genomic DNA was purified from blood and cultured skin fibroblasts using standard procedures. Mutations in mitochondrial ND1-6, ND4L and the $22 t R N A$ genes were ruled out. The coding exons and exon-intron boundaries of nuclear-encoded PUS1 and YARS2 genes were scanned by bidirectional Sanger sequencing using the BigDye Terminator v3.1 Cycle Sequencing Kit (Applied Biosystems, Foster City, CA, USA) on an ABI3130xl automatic DNA Analyzer.

Targeted resequencing was outsourced (BGI-Shenzhen, Shenzhen, China). A custom probe library was used for target enrichment (Agilent SureSelectXT Custom Kit) designed to capture coding exons and flanking intronic stretches (20 nt) of 1381 genes known to be functionally related to mitochondrial disorders ("Mitoexome”) (18), followed by deep sequencing using Illumina Hiseq technology (median reads depth $=255 X)$. Sanger sequencing was used to validate all the annotated functionally relevant variants, as well as well as to check variant segregation in the family.

\section{Lentiviral transduction}

The full length NDUFB 11 cDNA was amplified from a human RNA library using specific primers containing XbaI and XhoI restriction sites. The PCR amplified fragment was subcloned into the third generation lentivirus vector pcsc-SP-PW (pBOB) purchased from Addgene (Cambridge, MA, 
USA). Patient and control fibroblasts were transduced with a lentivirus containing either the wild type sequence of NDUFB11 or a GFP tag to assess the efficiency of the transduction (19).

\section{Results}

\section{Clinical, biochemical, and molecular findings}

The proband exhibited early-onset sideroblastic anemia in the absence of any motor or cognitive disability over time. Biochemical determination of MRC in muscle biopsy showed isolated complex I defect (-65\% complex I/citrate synthase of the lower control values). The biochemical defect of CI was also confirmed in fibroblasts mitochondria by measuring the rate of ATP synthesis, which was found to be significantly reduced $(-53 \%, p<0.001)$ compared to the control mean values, when malate was used as substrate, while normal rate values were obtained with succinate (Fig. 1a). In addition, the defect of complex I was also detected in fibroblasts using a dipstick enzyme activity assay, with activity at $23 \%$ of control values $(p<0.001)$ (Fig. S1a), while complex IV activity was normal (Fig. S1b). Accordingly, Western blotting of BNGE performed on fibroblast mitochondria, confirmed the dramatic reduction of the fully assembled CI (Fig. 1b). Western blotting on SDSPAGE of the single subunits of the MRC complexes showed a specific reduction of CI subunits, whereas the steady-state levels of subunits of the other MRC complexes were expressed within a normal range (Fig. 1c).

Initially genetic studies had excluded mtDNA pathogenic mutations in any of the CI structural subunits and the 22 tRNAs. Moreover, other candidate genes associated to MLASA, such as PUS1 and YARS2, were analyzed to exclude their putative involvement in the disease. The homozygous c.572G $>$ T [p.G191V] missense change identified in YARS2 was excluded to be pathogenic considering its high frequency of 0.13 (dbSNP, rs11539445) in normal populations (20). Parallel 
sequencing of a panel including 1381 genes, known to be associated with mitochondrial function, was performed. After excluding previously annotated single nucleotide changes occurring with high frequency in populations (frequency $>0.1 \%$ ), we prioritized variants predicted to have functional impact (i.e., nonsynonymous variants and changes affecting splice sites), taking into account a recessive inheritance model that, together with the maternal transmission, represent the most common inheritance models of mitochondrial disorders. This filtering led to the identification of a single gene entry, NDUFB11 (NC_000023.11, NM_019056.6), in which the hemizygous c.276_278delCTT was recognized. The variant was validated by Sanger sequencing and genotyping confirmed its de novo origin (Fig. 2a). The mutation had not been previously reported in public (dbSNP, ExAC) and in-house databases. The c.276_278delCTT mutation causes the loss of a phenylalanine residue $[\mathrm{p} .93 \mathrm{delF}]$ in a highly conserved region of the protein according to proteins multi alignment tools (ClustalW2). The impact of the mutation on the protein amount was deleterious resulting in a drastic reduction of NDUFB11 steady state level in patient's fibroblasts (Fig. 2b).

To further demonstrate that the biochemical defect observed in our patient was due to the identified NDUFB11 mutation, primary fibroblasts were transduced with wild type NDUFB11 by using a lentiviral vector. After transduction, the protein level of NDUFB11 in the patient increased considerably, when compared to untransduced cells or cells transduced with an empty vector (Fig. 2c). Western blotting of BNGE displayed a recovery in the amount of holocomplex I in the patient transduced with NDUFB11 of about $50 \%$ when compared to the ratio CI/CIII of the untransduced patient's cells. Accordingly, in-gel activity assay of BNGE showed a marked increase of CI activity in patient's cells transduced with NDUFB11 that reached almost control level (Figure 2d). These 
results proved evidence that the p.93delF variant in NDUFB11 was the cause of CI deficiency in the patient.

\section{Discussion}

We used targeted MitoExome analysis to characterize the genetic defect of a patient with early onset sideroblastic anemia and isolated complex I defect. We identified a novel c.276_278delCTT mutation in NDUFB11 predicting the loss of a phenylalanine [p.93delF], in a highly conserved region of the protein. The mutation is located in exon 2 upstream of the donor site of the alternative spliced transcript that gives rise to an mRNA 30 nucleotides longer than the canonical transcript. The shorter form is the most abundant and encodes for a protein that is located in the hydrophobic arm of complex I. To date, this is the third case reported with a mutation in $\operatorname{NDUFB11}(11,12)$ and, unlike the previously reported cases, our patient is a male and consequently carries the mutation in a hemizygous state.

Of note, the previously reported patients carried missense mutations or one base pair deletion and both lead to premature stop codon of the protein $(11,12)$. Conversely, our patient carried a three base pair deletion that did not cause the loss of the frame in the protein. This would point to a less dramatic impact of the mutation on protein stability. However, western blotting analysis of the steady state level of NDUFB11 showed a dramatic reduction of the protein that impacted on the activity and ultrastructure of the holocomplex I. Nevertheless some residual activity was still present as demonstrated by BNGE processed for in-gel activity assay and this was probably due to a partial functional protein which is still able to act inside the complex. These results are in line with previously reported data that still show a residual activity of CI in NDUFB11 silenced cells (11). However, unlike NDUFB11 silenced cells, we observed a marked reduction of other subunits of CI, 
including NDUFS1 and NDUFA9, located in the hydrophilic arm and at the junction between the hydrophobic and hydrophilic arm respectively $(21,22)$. These findings underline the importance of NDUFB11 not only for the correct assembly of the membrane arm but also for the assembly of the entire structure of CI. Indeed, reintroduction of NDUFB11 by lentiviral transduction restored the activity of $\mathrm{CI}$ in the patient demonstrating that complex I deficiency was due to the loss of this subunit.

Of note, all the reported cases harboring mutations in NDUFB11 show a peculiar phenotype which is rather uncommon for mitochondrial disorders. One report described two patients affected by a histiocytoid cardiomyopathy (12) while the second study reported two patients with microphthalmia and linear skin defect (11). The histiocytoid cardiomyopathy is a phenotype that has been already reported in relation to a mitochondrial gene inactivation $(23,24)$, as well as the linear skin defects with or without microphthalmia that has previously been associated with defects in two other mitochondrial genes, i.e. $C O X 7 B$ and $H C C S$, located on the $\mathrm{X}$ chromosome $(25,26)$. Our patient further expands the genetic and clinical phenotype associated with NDUFB11 defects.

Sideroblastic anemias are acquired or inherited anemias characterized by a decreased ability to synthesize hemoglobin in red blood cells and resulting in accumulation of iron deposits in the bone marrow eritroblasts that are called sideroblasts. This rare condition is caused by defects of genes involved in heme biosynthesis, iron-sulphur [Fe-S] cluster biosynthesis and mitochondrial protein synthesis $(27,28)$. The most common form of the X-linked sideroblastic anemia is due to erythroidspecific ' -aminolevulinate synthase (ALAS2) defect. Other known genes involved in sideroblastic anemia include the erythroid specific mitochondrial transporter (SLC25A38), adenosine triphosphate (ATP) binding cassette B7 ( $A B C B 7)$, glutaredoxin 5 (GLRX5), thiamine transporter (SLC19A2), the RNA-modifying enzyme pseudouridine synthase (PUS1), and mitochondrial 
tyrosyl-tRNA synthetase (YARS2), as well as mitochondrial DNA deletions. Recently, mutations in the mitochondrial encoded ATP6 gene, in the mitochondrial leucyl-transferase gene LARS2, in the tRNA nucleotidyl transferase TRNT1 and in HSPA9, a homologue of the mitochondrial HSP70, have been associated to sideroblastic anemia (29-33). Sideroblastic anemia can be associated to myopathy and lactic acidosis configuring the clinical picture of MLASA (Myopathy, Lactic Acidosis, Sideroblastic Anemia) and due to mutations in PUS1 (MLASA1, \#600462), YARS2 (MLASA2, \#613561) or MTATP6 (MLASA3, \#500011). It was also described associated to multisystem organ failure or immunodeficiency and developmental delay, caused respectively by LARS2 or TNRT1 mutations. To the best of our knowledge the patient here described is the first in whom a mutation in a mitochondrial complex I subunit is the cause of sideroblastic anemia as a new feature of a complex clinical phenotype.

Although mitochondrial diseases show a wide spectrum of phenotypes we cannot explain why different mutations in the same gene can cause such phenotypic divergence. NDUFB11 is highly expressed in heart tissue and this seems to be in line with our observation of fetal cardiac ventricular hypertrophy that, however, was spontaneously resolved in our patient. Morpholinomediated knockdown of $n d u f b 11$ in zebrafish embryos generated heart defects, with cardiomegaly, looping defects, and arrhythmia (12), however sideroblastic anemia was not observed in the $\mathrm{KO}$ ndufb11 zebrafish model. Conversely the gene is located in an X-chromosome region that is a hotspot for neurogenetic disorders (34), thus a hematologic phenotype is rather unexpected. Nevertheless, it is intriguing that a large number of genes involved in mitochondrial protein synthesis give rise to sideroblastic anemia.

In conclusion our studies have allowed the identification of a novel mutation in the NDUFB11 gene, associated to sideroblastic anemia and have widened the phenotypic and molecular spectrums 
associated to NDUFB11 deficiency. The development of appropriate animal models carrying single substitutions will probably help to better understand common pathways responsible for sideroblastic anemia in mitochondrial disorders and the pathogenic mechanisms underlining different clinical phenotypes related to defects in NDUFB11.

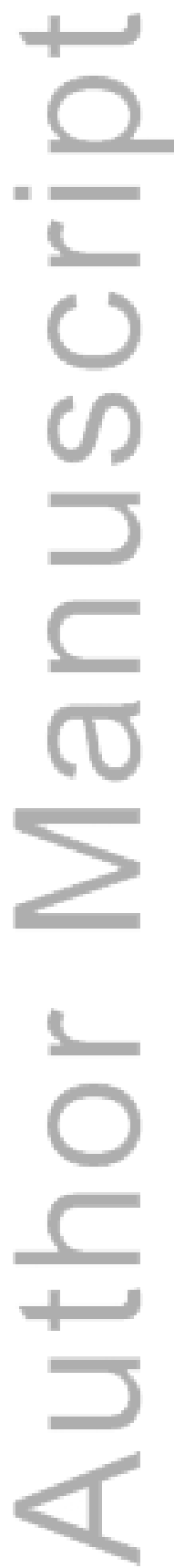

This article is protected by copyright. All rights reserved. 


\section{References}

1. Kirby DM, Crawford M, Cleary MA, Dahl HH, Dennett X, Thorburn DR. Respiratory chain complex I deficiency: an underdiagnosed energy generation disorder. Neurology 1999: 52 (6): $1255-1264$.

2. Carroll J, Shannon RJ, Fearnley IM, Walker JE, Hirst J. Definition of the nuclear encoded protein composition of bovine heart mitochondrial complex I. Identification of two new subunits. J Biol Chem 2002: 277 (52): 50311-50317.

3. Hatefi Y, Galante YM, Stiggal DL, Ragan CI. Proteins, polypeptides, prosthetic groups and enzymic properties of complexes I, II, III, IV, and V of the mitochondrial oxidative phosphorylation system. Methods Enzymol 1979: 56: 577-602.

4. Hatefi Y: The mitochondrial electron transport and oxidative phosphorylation system. Ann Rev Biochem 1985; 54: 1015-1069.

5. Koene S, Rodenburg RJ, van der Knaap MS et al. Natural disease course and genotypephenotype correlations in Complex I deficiency caused by nuclear gene defects: what we learned from 130 cases. J Inherit Metab Dis 2012: 35 (5): 737-747.

6. Pagniez-Mammeri H, Loublier S, Legrand A, Bénit P, Rustin P, Slama A. Mitochondrial complex I deficiency of nuclear origin I. Structural genes. Mol Genet Metab 2012: 105 (2):

\section{$163-172$.}

7. Hoefs SJ, Rodenburg RJ, Smeitink JA, van den Heuvel LP. Molecular base of biochemical complex I deficiency. Mitochondrion 2012: 12 (5): 520-532.

8. Mimaki M, Wang X, McKenzie M, Thorburn DR, Ryan MT. Understanding mitochondrial complex I assembly in health and disease. Biochim Biophys Acta 2012: 1817 (6): 851-862. 
9. Pagniez-Mammeri H, Rak M, Legrand A, Bénit P, Rustin P, Slama A. Mitochondrial complex I deficiency of nuclear origin II. Non-structural genes. Mol Genet Metab 2012: 105 (2): 173-179.

10. Finsterer J, Frank M. Haematological abnormalities in mitochondrial disorders. Singapore Med J 2015: 56 (7): 412-419.

11. van Rahden VA, Fernandez-Vizarra E, Alawi M et al. Mutations in NDUFB11, encoding a complex I component of the mitochondrial respiratory chain, cause microphthalmia with linear skin defects syndrome. Am J Hum Genet 2015: 96 (4): 640-650.

12. Shehata BM, Cundiff CA, Lee $\mathrm{K}$ et al. Exome sequencing of patients with histiocytoid cardiomyopathy reveals a de novo NDUFB11 mutation that plays a role in the pathogenesis of histiocytoid cardiomyopathy. Am J Med Genet A 2015: 167 (9): 2114-2121.

13. Potluri P, Yadava N, Scheffler IE. The role of the ESSS protein in the assembly of a functional and stable mammalian mitochondrial complex I (NADH-ubiquinone oxidoreductase). Eur J Biochem 2004: 271 (15): 3265-3273.

14. Bugiani M, Invernizzi F, Alberio S et al. Clinical and molecular findings in children with complex I deficiency. Biochim Biophys Acta 2004: 1659 (2-3): 136-147.

15. Rizza T, Vazquez-Memije ME, Meschini MC et al. Assaying ATP synthesis in cultured cells: a valuable tool for the diagnosis of patients with mitochondrial disorders. Biochem Biophys Res Commun 2009: 383 (1): 58-62.

16. Nijtmans LG, Henderson NS, Holt IJ. Blue native electrophoresis to study mitochondrial and other protein complexes. Methods 2002: 26 (4): 327-334. 
17. Zerbetto E, Vergani L, Dabbeni-Sala F. Quantification of muscle mitochondrial oxidative phosphorylation enzymes via histochemical staining of blue native polyacrylamide gels. Electrophoresis 1997: 18 (11): 2059-2064.

18. Calvo SE, Compton AG, Hershman SG et al. Molecular diagnosis of infantile mitochondrial disease with targeted next-generation sequencing. Sci Transl Med 2012: 4 (118): 118ra10.

19. Naldini L and Verma IM. Lentiviral vectors. Adv Virus Res 2000: 55: 599-609.

20. Riley LG, Menezes MJ, Rudinger-Thirion J et al. Phenotypic variability and identification of novel YARS2 mutations in YARS2 mitochondrial myopathy, lactic acidosis and sideroblastic anaemia. Orphanet J Rare Dis 2013: 8: 193.

21. Sazanov LA, Baradaran R, Efremov RG, Berrisford JM, Minhas G. A long road towards the structure of respiratory complex I, a giant molecular proton pump. Biochem Soc Trans 2013: 41 (5): 1265-1271.

22. Stroud DA, Formosa LE, Wijeyeratne XW, Nguyen TN, Ryan MT. Gene knockout using transcription activator-like effector nucleases (TALENs) reveals that human NDUFA9 protein is essential for stabilizing the junction between membrane and matrix arms of complex I. J Biol Chem 2013; 288 (3): 1685-1690.

23. Andreu AL, Checcarelli N, Iwata S, Shanske S, DiMauro S. A missense mutation in the mitochondrial cytochrome $\mathrm{b}$ gene in a revisited case with histiocytoid cardiomyopathy. Pediatr Res 2000: 48 (3): 311-314.

24. Götz A, Tyynismaa H, Euro L et al. Exome sequencing identifies mitochondrial alanyltRNA synthetase mutations in infantile mitochondrial cardiomyopathy. Am J Hum Genet 2011: 88 (5): 635-642.

This article is protected by copyright. All rights reserved. 
25. Wimplinger I, Morleo M, Rosenberger $G$ et al. Mutations of the mitochondrial holocytochrome c-type synthase in X-linked dominant microphthalmia with linear skin defects syndrome. Am J Hum Genet 2006: 79 (5): 878-889.

26. Indrieri A, van Rahden VA, Tiranti V et al. Mutations in COX7B cause microphthalmia with linear skin lesions, an unconventional mitochondrial disease. Am J Hum Genet 2012: $91(5): 942-949$.

27. Fujiwara T, Harigae H. Pathophysiology and genetic mutations in congenital sideroblastic anemia. Pediatr Int 2013: 55 (6):675-679.

28. Bottomley SS, Fleming MD. Sideroblastic anemia: diagnosis and management. Hematol Oncol Clin North Am 2014: 28 (4):653-670.

29. Burrage LC, Tang S, Wang J et al. Mitochondrial myopathy, lactic acidosis, and sideroblastic anemia (MLASA) plus associated with a novel de novo mutation (m.8969G $>$ A) in the mitochondrial encoded ATP6 gene. Mol Genet Metab 2014: 113 (3): 207-212.

30. Riley LG, Rudinger-Thirion J, Schmitz-Abe K et al. LARS2 Variants Associated with Hydrops, Lactic Acidosis, Sideroblastic Anemia, and Multisystem Failure. JIMD Rep 2015; e-pub ahead of print 5 November 2015.

31. Chakraborty PK, Schmitz-Abe K, Kennedy EK et al. Mutations in TRNT1 cause congenital sideroblastic anemia with immunodeficiency, fevers, and developmental delay (SIFD). Blood 2014: 124 (18): 2867-2871.

32. Sasarman F, Thiffault I, Weraarpachai $\mathrm{W}$ et al. The 3' addition of CCA to mitochondrial tRNASer(AGY) is specifically impaired in patients with mutations in the tRNA nucleotidyl transferase TRNT1. Hum Mol Genet 2015: 24 (10): 2841-2847. 
33. Schmitz-Abe K, Ciesielski SJ, Schmidt PJ et al. Congenital sideroblastic anemia due to mutations in the mitochondrial HSP70 homologue HSPA9. Blood 2015: 126 (25):27342738.

34. Thiselton DL, McDowall J, Brandau O et al. An integrated, functionally annotated gene map of the DXS8026-ELK1 interval on human Xp11.3-Xp11.23: potential hotspot for neurogenetic disorders. Genomics 2002: 79 (4): 560-572.

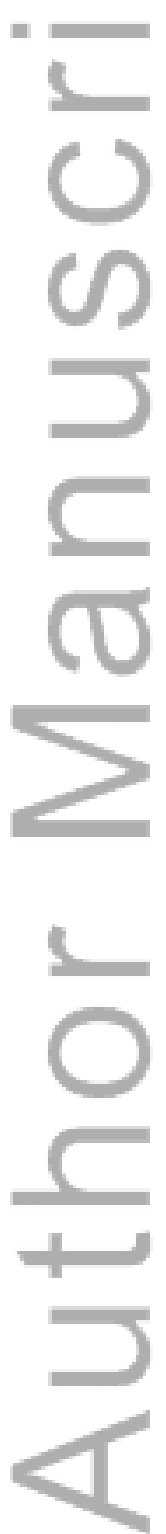

This article is protected by copyright. All rights reserved. 


\section{Figure Legends}

Fig. 1. Biochemical and structural analyses in patient's fibroblasts. (a) Spectrophotometric determination of complex $V$ activity. The rate of ATP synthesis in fibroblasts mitochondria was reduced of $53 \%(p<0.001)$ compared to the controls mean value when malate $(\mathrm{M})$ was used as substrate to energize mitochondria, whereas a normal value was obtained with succinate (S); (b) Blue Native Gel Electrophoresis. Western blotting of BNGE was performed on mitochondria isolated from fibroblasts. Specific antibodies against complex I (CI) (NDUFA9), complex III (CIII) (UQCRC2), complex IV (CIV) (COXI) and complex II (CII) (SDHB) confirmed the dramatic and isolated reduction of complex I. (c) Western blotting analysis on SDS gel. Patient's (Pt) derived mitochondria displays a specific reduction of the complex I subunits (NDUFS1 and NDUFA9) compared to controls (C1, C2); whereas the subunits of complex II (SDHB), complex III (UQCRC2), and complex IV (COXII) are normally expressed. The mitochondrial protein porin (VDAC) was used as a control for equal loading.

\section{Fig. 2. Genetic features and lentiviral transfection of patient's fibroblasts with wild-type} NDUFB11. (a) Electropherograms. The genomic region of the patient (Pt), showing the deletion in exon 2 of NDUFB11, and its Mother (Mo) it is shown. (b) Western blotting on SDS-PAGE. The mutation produced a marked reduction of the steady state level of the corresponding protein in the patient. (c) SDS-PAGE on transduced patient's fibroblasts. Infected sample shows a marked increase in the level of NDUFB11 protein compared to untransduced cells $(\mathrm{Pt})$ or patient cells transduced with empty vector $(\mathrm{Pt}+\mathrm{EV})$. (d) BNGE on transduced patient's fibroblasts. In-gel activity (IGA) assay showed a marked increase of CI activity in cells transduced with a wild type copy of NDUFB11, compared to the undetectable activity in untransduced cells. In addition, a 
recovery in the amount of holocomplex I in the same sample is evident. The amount of complex III (CIII) is normal.

Fig. S1. Dipstick enzyme assay of complex I and IV activity. In patient fibroblasts (Pt) complex I activity was at $23 \%(p<0.001)$ of controls $(\mathrm{Ct})(\mathrm{a})$. Complex IV displayed a normal activity (b).

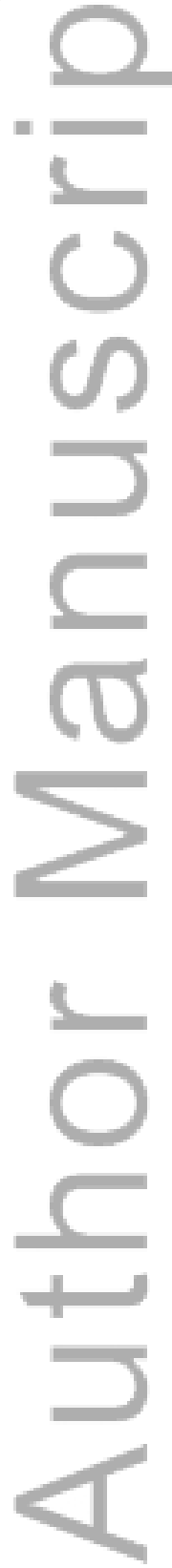

This article is protected by copyright. All rights reserved. 


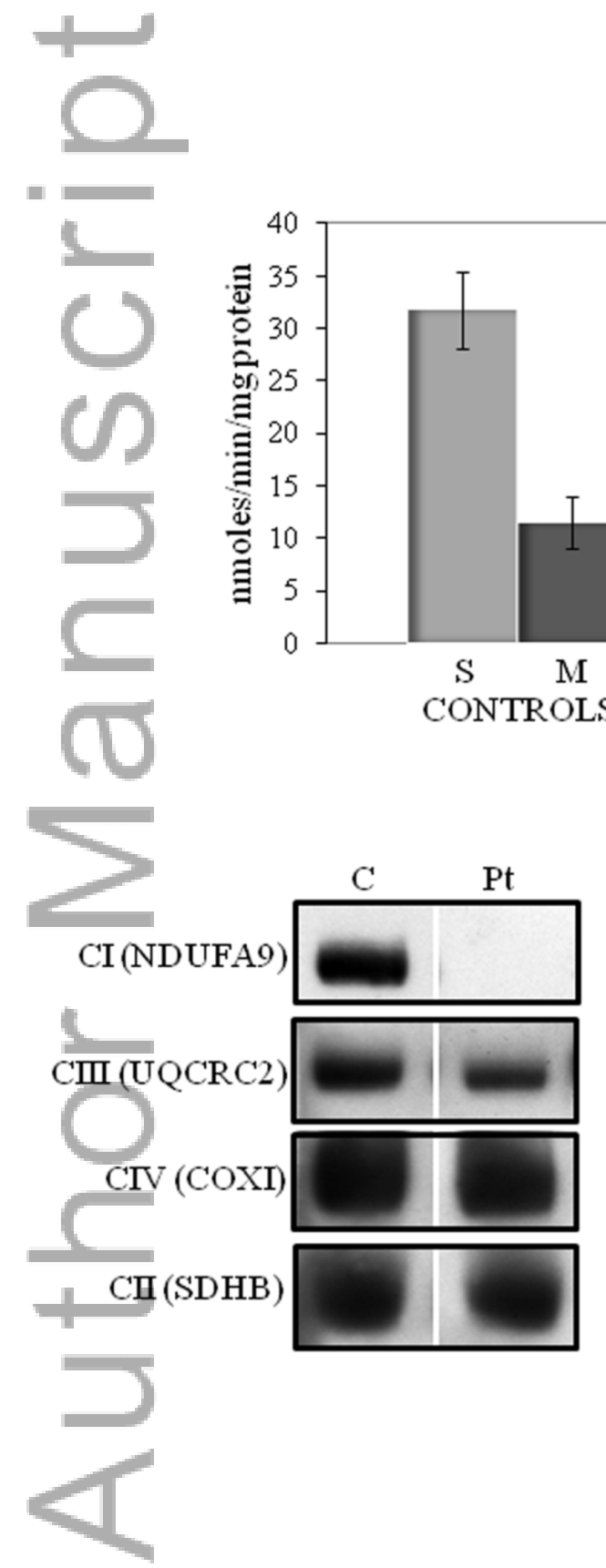

(b)

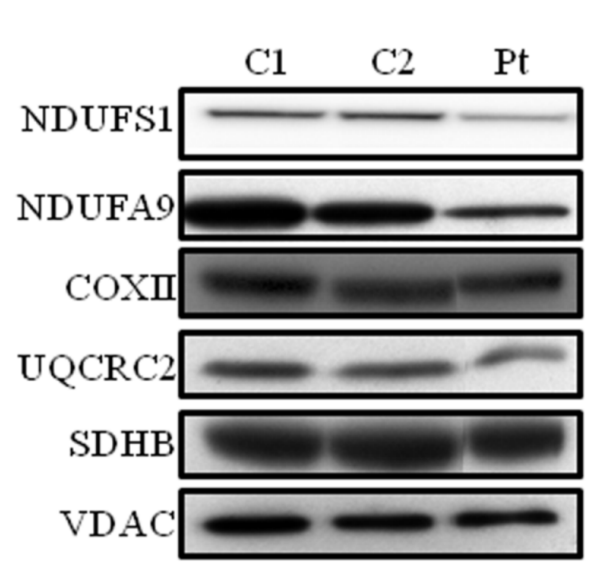

(c)

Fig. 1.tif 


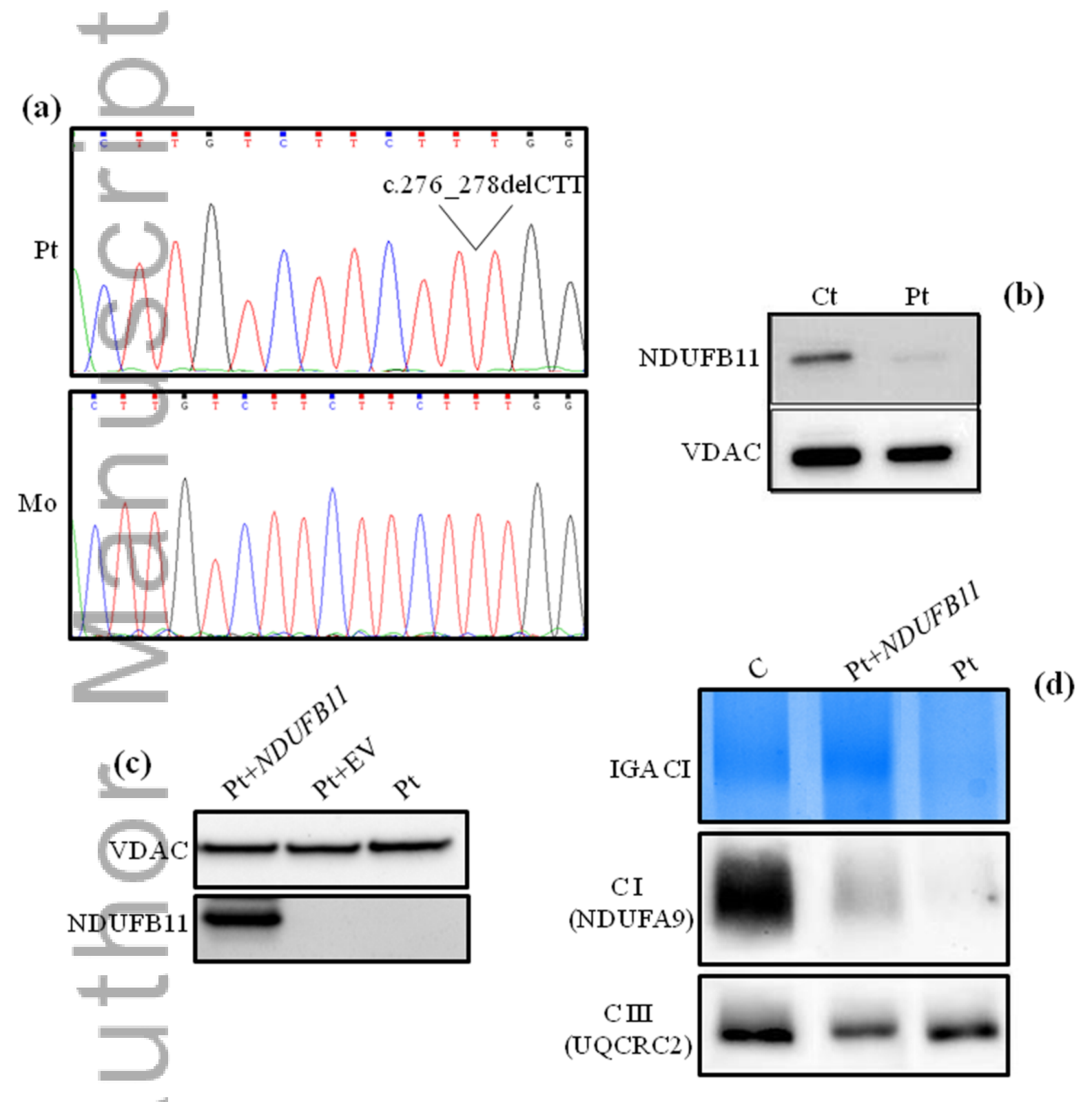

Fig. 2.tif

This article is protected by copyright. All rights reserved. 


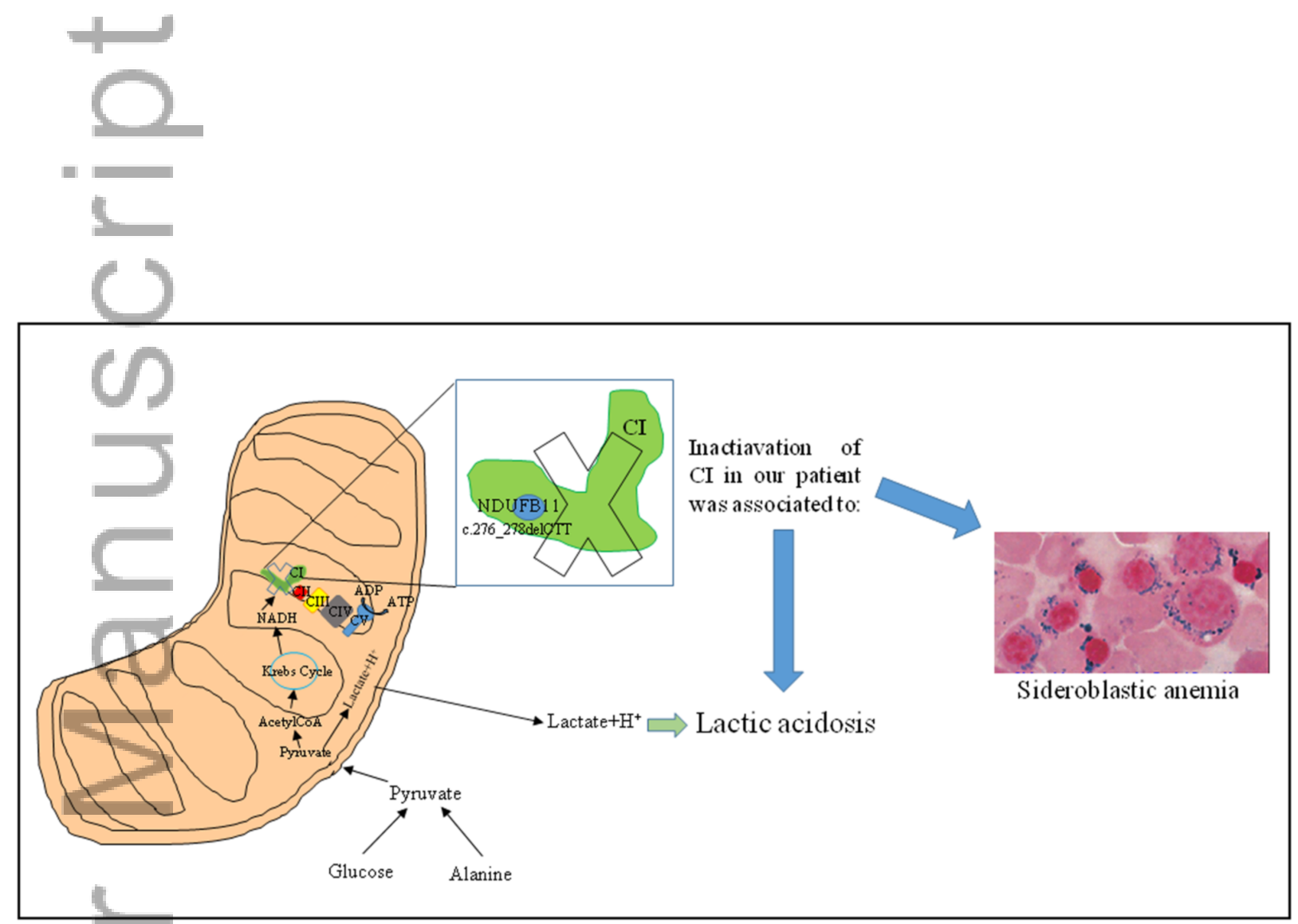

Graphical Abstract: Inactivation of CI due to a de-novo mutation in NDUFB1l gene, encoding for a small and hydrophobic subunit of CI, produces a peculiar phenotype in our patient characterized by lactic acidosis and sideroblastic anemia.

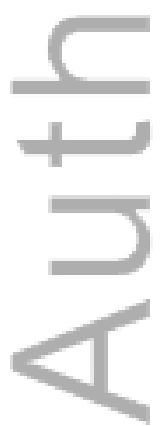

\section{Graphical Abstract.tif}

This article is protected by copyright. All rights reserved. 


\section{University Library}

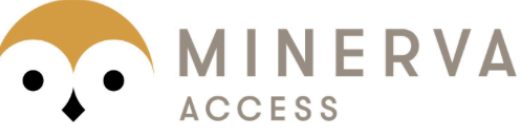

A gateway to Melbourne's research publications

Minerva Access is the Institutional Repository of The University of Melbourne

\section{Author/s:}

Torraco, A;Bianchi, M;Verrigni, D;Gelmetti, V;Riley, L;Niceta, M;Martinelli, D;Montanari, A;Guo, Y;Rizza, T;Diodato, D;Di Nottia, M;Lucarelli, B;Sorrentino, F;Piemonte, F;Francisci, S;Tartaglia, M;Valente, EM;Dionisi-Vici, C;Christodoulou, J;Bertini, E;Carrozzo, R

Title:

A novel mutation in NDUFB11 unveils a new clinical phenotype associated with lactic acidosis and sideroblastic anemia

\section{Date:}

2017-03-01

\section{Citation:}

Torraco, A., Bianchi, M., Verrigni, D., Gelmetti, V., Riley, L., Niceta, M., Martinelli, D., Montanari, A., Guo, Y., Rizza, T., Diodato, D., Di Nottia, M., Lucarelli, B., Sorrentino, F., Piemonte, F., Francisci, S., Tartaglia, M., Valente, E. M., Dionisi-Vici, C. ,... Carrozzo, R. (2017). A novel mutation in NDUFB11 unveils a new clinical phenotype associated with lactic acidosis and sideroblastic anemia. CLINICAL GENETICS, 91 (3), pp.441-447. https:// doi.org/10.1111/cge.12790.

Persistent Link:

http://hdl.handle.net/11343/291345 